\title{
2019 ERS cough guideline: consensus and controversy
}

\author{
Ruchong Chen ${ }^{1}$, Zhongmin Qiu ${ }^{2}$, Kefang Lai ${ }^{1}$ \\ ${ }^{1}$ National Clinical Research Center for Respiratory Disease, State Key Laboratory of Respiratory Disease, Guangzhou Institute of Respiratory \\ Health, the First Affiliated Hospital of Guangzhou Medical University, Guangzhou, China; ${ }^{2}$ Department of Pulmonary and Critical Care Medicine, \\ Tongji Hospital, Tongii University School of Medicine, Shanghai, China \\ Correspondence to: Prof. Zhongmin Qiu. Department of Pulmonary and Critical Care Medicine, Tongji Hospital, Tongji University School of \\ Medicine, No. 389 Xincun Road, Shanghai 200065, China. Email: qiuzhongmin@tongji.edu.cn; Prof. Kefang Lai. State Key Laboratory of \\ Respiratory Disease, National Clinical Research Center for Respiratory Disease, Guangzhou Institute of Respiratory Health, the First Affiliated \\ Hospital of Guangzhou Medical University, No. 151 Yanjiang Road, Guangzhou 510120, China. Email: klai@163.com.
}

Submitted Oct 15, 2020. Accepted for publication Dec 15, 2020.

doi: $10.21037 /$ jtd-2020-065

View this article at: http://dx.doi.org/10.21037/jtd-2020-065

In the beginning of 2020, the European Respiratory fournal published ERS guidelines on the diagnosis and treatment of chronic cough in adults and children. The European Respiratory Society (ERS) first published the guideline for the diagnosis and treatment of cough in 2004. Subsequently, the American College of Chest Physicians (ACCP) guideline and Chinese Thoracic Society (CTS) guideline was published in 2006. The guidelines of the assessment of cough and the Expert opinion on the cough hypersensitivity syndrome by ERS was launched in 2007 and 2014, respectively. All of the above mentioned played an important role in the improvement of management and research in cough. To improve diagnostic accuracy and promote evidence-based therapy for patients with chronic cough in primary and secondary care, the ERS Task Force (TF) chaired by Prof. A. H. Morice and Prof. E. Millqvist started to update the guideline in 2017, and released it at the European Respiratory Annual Congress in October 2019. The TF consisted of multidisciplinary experts from European countries (the United Kingdom, Sweden, the Netherlands, Switzerland, Spain etc.), as well as of three specialists from United States, China, and Korea. Here, we attempted to lay out the key points of the updated guideline and summarize the consensus and controversy regarding the current issues (Table 1).

\section{Methodology and structure}

The updated guideline was set up following the methodology recommended by the ERS Guidelines Working Group and Science Council. It not only highlighted the evidencebased graded recommendations for eight key questions via a systematical GRADE framework, but also provided systematic reviews on some clinically important aspects of chronic cough (definition, epidemiology, impact on patients, etiology and mechanisms, clinical phenotypes, chronic cough in children, refractory cough, tobacco exposure, assessing and treatment, and future perspectives). Meanwhile, the newly edited guideline offered two flow charts, which benefits the clinical practitioners to assess cough of adults and children.

The Task Force members collected a series of important issues relevant to the management of chronic cough. According to the PICO (Population, Intervention, Comparison and Outcomes) processing, eight questions of clinical uncertainty (two diagnostic and six therapeutic questions) were generated. Aimed at each PICO question, systematic reviews of related literature were identified by searching Pubmed/MEDLINE, Embase, and Cochrane Central Register of Controlled Trials databases until June 2018. The recommendations to each question were proposed via evidence-based GRADE (Grading of Recommendations Assessment, Development and Evaluation) approaches. The research protocols including the searching strategy and data analysis are available in the online supplement.

\section{Key questions, recommendations and comments}

Question 1: Should chest CT scan be routinely performed on chronic cough patients with normal chest $X$-ray and physical examination?

Recommendation 1: We suggest that clinicians should not routinely perform a chest CT scan in patients with 
Table 1 Comparison of guideline recommendation in different chronic cough guidelines

\begin{tabular}{|c|c|c|}
\hline ERJ & ACCP & CTS \\
\hline \multicolumn{3}{|c|}{ Question 1: Should chest CT scan be routinely performed on chronic cough patients with normal chest X-ray and physical examination? } \\
\hline $\begin{array}{l}\text { Recommendation } 1 \text { : We suggest that } \\
\text { clinicians do not routinely perform a } \\
\text { chest CT scan in patients with chronic } \\
\text { cough who have a normal chest X-ray } \\
\text { and physical examination. (conditional } \\
\text { recommendation, very low-quality } \\
\text { evidence) }\end{array}$ & $\begin{array}{l}\text { In the flowchart, CT, as a further investigation, is } \\
\text { recommended for the patients with chronic cough who } \\
\text { have inadequate response to optimal treatment after } \\
4-6 \text { weeks follow up (1) }\end{array}$ & $\begin{array}{l}\text { Chest radiographs are routinely recommended for chronic } \\
\text { cough (Grade 2D). If an obvious abnormality is observed on } \\
\text { plain films, additional investigation is selected based on the } \\
\text { characteristics of the lesion. High-resolution CT is helpful } \\
\text { for the early diagnosis of interstitial pulmonary diseases and } \\
\text { atypical bronchiectasis }\end{array}$ \\
\hline
\end{tabular}

Question 2: Should FeNO/blood eosinophils be used to predict treatment response to corticosteroids/anti-leukotrienes in chronic cough?

Research recommendation

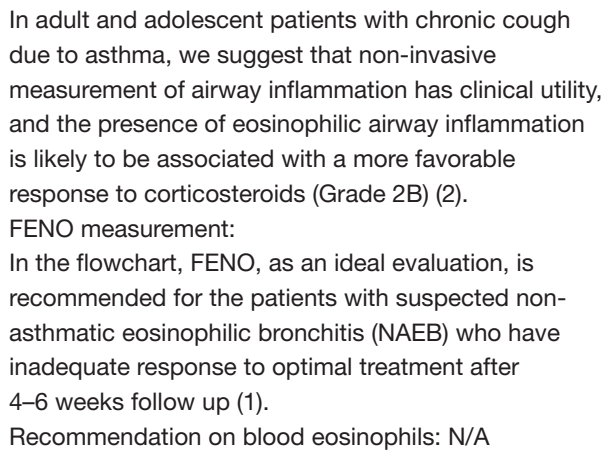

(I) Recommendation on FeNO measurement:

(i) An increase in FeNO (>32 ppb) suggests eosinophilic inflammation or corticoid-sensitive cough. However, the sensitivity is not high when FeNO measurement is used for screening of eosinophilic inflammation. Approximately $40 \%$ of patients with increased numbers of eosinophils have normal FeNO (Grade 2C).

(ii) The measurement of $\mathrm{FeNO}$ is recommended as to supplement of the induced sputum test (Grade 2C).

(iii) The sensitivity of FeNO is not high for diagnosis of $\mathrm{EB}$, but FeNO of $>32$ ppb suggests eosinophil-related chronic cough (e.g., EB or CVA) (Grade 2C)

(II) Recommendation on blood eosinophils: N/A

Question 3: Should anti-asthmatic drugs (anti-inflammatory or bronchodilator drugs) be used to treat patients with chronic cough?

\begin{abstract}
Recommendation 3a: We conditiona low suggest a short-term ICS trial (2-4 weeks) in adult patients with chronic cough. (conditional recommendation, low quality evidence)

Recommendation 3b: We suggest a short-term ICS trial (2-4 weeks) in children with chronic dry cough. (conditional recommendation, low quality evidence)

Recommendation 3c: We suggest a short-term anti- leukotriene trial (24 weeks) in adults with chronic cough, particularly for those with asthmatic cough. (conditional recommendation, low quality evidence)

Recommendation 3d: We suggest a short-term trial (2-4 weeks) of ICS and long-acting bronchodilator combination in adults with chronic cough and fixed airflow obstruction. (conditional recommendation, moderate quality evidence)
\end{abstract}

(I) In adult and adolescent patients with chronic cough due to asthma as a unique symptom (cough variant asthma [CVA]) we suggest that inhaled corticosteroids should be considered as first line treatment. If response is incomplete in those with CVA or if cough is the remaining isolated symptom following treatment with inhaled corticosteroids in patients with asthma in whom cough was one of their symptoms we suggest stepping-up the inhaled corticosteroid dose and considering a therapeutic trial of a leukotriene inhibitor after reconsideration of alternative causes of cough. Beta-agonists could also be considered in combination with ICS. (Grade1B) (2).

(II) In adult and adolescent patients with chronic cough due to NAEB, we suggest inhaled corticosteroids as firstchoice treatment (Grade 2B) (2).

(III) In adult and adolescent patients with chronic cough due to NAEB if response to ICS is incomplete, we suggest stepping-up the inhaled corticosteroid dose and considering a therapeutic trial of a leukotriene inhibitor after reconsideration of alternative causes of cough (Grade 2C) (2).

(IV) In adult patients with chronic cough, we suggest that patients with chronic cough undergo a guideline/protocol based assessment process that includes objective testing for bronchial hyperresponsiveness and eosinophilic bronchitis, or a therapeutic corticosteroid trial (Ungraded Consensus-Based Statement) (3)

\section{(I) Recommendation on CVA:}

(i) A combination of ICS and bronchodilator ( $\beta_{2}$ receptor agonist), such as budesonide/formoterol and fluticasone/ formoterol, is recommended (Grade 1B).

(ii) The treatment should last for more than 8 weeks, and in some patients, long-term treatment may be required (Grade 2D).

(iii) A short-term oral corticosteroid (10-20 mg/d, for 3-5 days) is recommended for patients refractory or less responsive to ICS treatment, or in patients suffering from severe inflammation of the airway (Grade 2C).

(iv) Long-term use of ICS may be helpful to prevent development into typical asthma (Grade 2B).

(v) Leukotriene receptor antagonists are effective in improving cough, airway inflammation, and the quality of life (Grade 2B)

(II) Recommendation on EB:

The use of ICS is the first therapeutic option, and treatment course with more than 8 weeks is recommended (Grade 2C). (III) Recommendation on AC:

The treatment course of ICS should last for more than 4 weeks, and oral corticosteroids can be used initially for a short period (3-5 days) for treatment (Grade 2C).

(IV) Recommendation on empirical management of chronic cough:

If steroid-responsive cough is suspected, oral administration of low-dose steroids for 1 week is recommended, followed by ICS or combined therapy with $\beta 2$-receptor agonists (Grade 2C)

Table 1 (continued) 
Table 1 (continued)

\begin{tabular}{ll}
\hline ERJ & ACCP \\
\hline Question 4: Should anti-acid drugs (PPIs and H2 antagonists) be used to treat patients with chronic & \\
$\begin{array}{l}\text { Recommendation 4: We suggest } \\
\text { that clinicians do not routinely use }\end{array}$ & (I) In adult patients with chronic cough suspected to \\
anti-acid drugs in adult patients & be due to reflux-cough syndrome, we recommend \\
with chronic cough. (conditional & weight loss in overweight or obese patients; (ii) head \\
recommendation, low quality & of bed elevation and avoiding meals within 3 hours of \\
evidence) & bedtime; and (iii) in patients who report heartburn and \\
& regurgitation, proton pump inhibitors, H2- receptor \\
& antagonists, alginate, or antacid therapy sufficient to \\
& control these symptoms (Grade 1C) (4). \\
& (II) In adult patients with suspected chronic cough \\
& due to reflux-cough syndrome, but without heartburn \\
& or regurgitation, we recommend against using proton \\
& pump inhibitor therapy alone because it is unlikely to \\
be effective in resolving the cough (Grade 1C) (4). \\
(III) In adult patients with unexplained chronic cough \\
and a negative workup for acid gastroesophageal \\
reflux disease, we suggest that proton pump inhibitor \\
therapy not be prescribed (2C) (3)
\end{tabular}

CTS

Question 5: Should drugs with pro-motility activity (reflux inhibitors, prokinetics and macrolides with pro-motility activity) be used to treat patients with chronic cough?

Recommendation 5: There is currently Remarks: insufficient evidence to recommend the routine use of macrolide therapy in chronic cough. A one-month trial of macrolides can be considered in the cough of chronic bronchitis refractory to other therapy, taking into account local guidelines on antimicrobial stewardship. (conditional recommendation, low quality evidence)
In the updated 2016 guideline, it showed that with respect to the treatment arms, they were all placebo controlled with respect to the primary intervention (PPIs in 11 of 14 cases), but most of the trials made no mention of concomitant dietary modifications, lifestyle modifications, or use of prokinetic drugs (4). Due to lack of sufficient evidence, the use of prokinetic drugs al could not be concluded as a recommendation to treat patients with chronic cough. However, in the 2006 guideline, the prokinetic therapy was recommended in treating some patients with cough due to GERD. The detailed recommendation in the 2006 guideline was showed as below. In some patients, cough due to GERD will favorably respond to acid suppression therapy alone; proton pump inhibition may be effective when $\mathrm{H} 2$-antagonism has been ineffective; prokinetic therapy and diet, when added to proton pump inhibition, may be effective when proton pump inhibition alone has been ineffective (Level of evidence, low; benefit, substantial; grade of recommendation, B) (5)
Recommendation on prokinetic agents: Most patients with GERC have esophageal motility dysfunction, therefore the addition of prokinetic agents (domperidone and mosapride) is recommended (Grade 1D)
Proton pump inhibitor (PPI) is recommended for patients suspected to be due to GERC (Grade 2C).

Antacids: acid suppression is recommended as the standard treatment for GERC (Grade 1A).

When the treatment with the standard dose of PPI is not effective, increasing the dose of PPI may be helpful (Grade 2A).

If treatment with one kind of PPI fails, switching to another PPI may be effective (Grade 2C).

Combining $\mathrm{H}_{2}$ receptor antagonist with PPI may ameliorate cough symptoms due to refractory gastroesophageal reflux or nighttime acid reflux (Grade 2C)

(dom

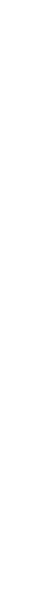

\footnotetext{
Question 6: Which cough neuromodulatory agents (pregabalin, gabapentin, tricyclics, and opiates) should be used to treat patients with chronic cough?

Recommendation 6a: We recommend a In adult patients with unexplained chronic cough, we trial of low dose morphine (5-10 mg bd) suggest a therapeutic trial of gabapentin as long as the in adult patients with chronic refractory potential side effects and the risk-benefit profile are cough. (strong recommendation, moderate quality evidence) Recommendation 6b: We suggest a trial of gabapentin or pregabalin in adult patients with chronic refractory cough. (conditional recommendation, low quality evidence)

Clinical studies show that neuromodulators, for example gabapentin, are effective in the treatment of $\mathrm{CHS}$ (Grade 2B). Other medicines including amitriptyline, baclofen, carbamazepine, and pregabalin may be useful in $\mathrm{CHS}$ (Grade 2C). and there is a reassessment of the risk- benefit profile at 6 months before continuing the drug (Grade 2C) (3) Dextromethorphan are recommended for chronic cough in adults (Grade 2A). Refractory GERC can be treated with baclofen. Adverse effects of baclofen include drowsiness and fatigue (Grade 2C)
}

Table 1 (continued) 
Table 1 (continued)

\begin{tabular}{|c|c|c|}
\hline ERJ & ACCP & CTS \\
\hline \multicolumn{3}{|c|}{ Question 7: Should non-pharmacological therapy (cough control therapy) be used to treat patients with chronic cough? } \\
\hline $\begin{array}{l}\text { Recommendation } 7 \text { : We suggest } \\
\text { a trial of cough control therapy in } \\
\text { adult patients with chronic cough. } \\
\text { (conditional recommendation, } \\
\text { moderate quality evidence) }\end{array}$ & $\begin{array}{l}\text { In adult patients with unexplained chronic cough, we } \\
\text { suggest a therapeutic trial of multimodality speech } \\
\text { pathology therapy (Grade } 2 \text { C) (3) }\end{array}$ & $\begin{array}{l}\text { Other potential treatments including speech therapy, and } \\
\text { cough suppression physical therapy. Cough suppression } \\
\text { physical therapy improves the quality of life related to } \\
\text { cough, cough hypersensitivity, and cough frequency } \\
\text { (Grade 2B) }\end{array}$ \\
\hline
\end{tabular}

Question 8: Should a trial of antibiotics be used in children with chronic wet cough with normal chest X-ray, normal spirometry and no warning signs?

\begin{tabular}{|c|c|}
\hline $\begin{array}{l}\text { Recommendation 8: We suggest a trial } \\
\text { of antibiotics in children with chronic } \\
\text { wet cough with normal chest X-rays, } \\
\text { normal spirometry, and no warning } \\
\text { signs. (conditional recommendation, } \\
\text { low quality evidence) }\end{array}$ & $\begin{array}{l}\text { (I) For children aged } \leq 14 \text {-year with chronic } \\
\text { ( } 4 \text {-week duration) wet or productive cough } \\
\text { unrelated to an underlying disease and without any } \\
\text { other specific cough pointers (e.g., coughing with } \\
\text { feeding, digital clubbing), we recommend } 2 \text {-week of } \\
\text { antibiotics targeted to common respiratory bacteria } \\
\text { (Streptococcus pneumoniae, Haemophilus influenzae, } \\
\text { Moraxella catarrhalis) targeted to local antibiotic } \\
\text { sensitivities (Grade } 1 \text { A) (6). } \\
\text { (II) For children aged } \leq 14 \text {-year with chronic ( }>4 \text {-week } \\
\text { duration) wet or productive cough unrelated to an } \\
\text { underlying disease and without any other specific } \\
\text { cough pointers (e.g., coughing with feeding, digital } \\
\text { clubbing), when the wet cough persists after } 2 \text {-week of } \\
\text { appropriate antibiotics, we recommend treatment with } \\
\text { an additional 2-week of the appropriate antibiotic(s) } \\
\text { (Grade 1C) (6) }\end{array}$ \\
\hline
\end{tabular}

ACCP, American College of Chest Physicians; BPC, bronchoprovocation challenge; CTS, Chinese Thoracic Society; CT, computed tomography; ERS, European Respiratory Society; FeNO, fractional exhaled nitric oxide; ICS, inhaled corticosteroids; CHS, cough hypersensitivity syndrome; NAEB, nonasthmatic eosinophilic bronchitis; PBB, protracted bacterial bronchitis.

chronic cough who have normal chest X-ray and physical examination (conditional recommendation, very low-quality evidence).

Comments: In the ERS guidelines, chest CT is not recommended as a first-line routine examination in subjects with normal chest $\mathrm{X}$-ray, which is consistent with the recommendations of ACCP and CTS. The balance between clinical benefits and potential risk from CT radiation exposure needs to be weighed, while the benefits of CT examination lack the high-quality evidence. There is a concern about potential risks of radiation in susceptible populations such as children and females. For the majority of patients with normal chest X-ray, there are no obvious abnormalities in CT findings, and abnormal CT findings do not necessarily indicate the cause of cough (7). Thus, repeated chest radiographs, especially CT scan, is not useful for diagnosis. Furthermore, it may increase the medical expense and the potential cancer risk (8-10). Thus, in terms of the overuse of CT scanning in clinical practice, this
The etiologic distribution of chronic cough in children is not identical to that in adults, and varies with age. For children younger than 3 years of age, airway infection should be initially considered (Grade 2C) recommendation is necessary.

However, for the refractory cough patients, after thorough evaluation, clinicians can consider chest CT scan, and even a bronchoscopy evaluation in order to avoid missing diagnosis of some rare causes $(1,11)$. In some subjects with rare or uncommon causes of chronic cough, obvious abnormalities may not be found by chest $\mathrm{X}$-rays. But if cough persists after consideration of the most common causes, a chest CT scan may be useful to identify some uncommon conditions, including earlystaged bronchial tumors, simple bronchial tuberculosis, bronchial foreign bodies, bronchial amyloidosis, etc. (11). If common causes of chronic cough have been excluded (negative routine chronic cough examinations or ineffective empirical treatment), performing chest CT examination may be necessary to avoid missing out some rare causes of chronic cough, and it can be combined with medical history and physical examination to improve the accuracy of diagnosis. 
Question 2: Should FeNO/blood eosinophils be used to predict treatment response to corticosteroids/antileukotrienes in chronic cough?

Recommendation 2: Convenient and practical tests are needed for predicting anti-inflammatory treatment responses in patients with chronic cough. However, there is a still lack of quality evidence. Placebo-controlled trials are warranted to assess their utility and also consensus is required on threshold levels in patients with chronic cough. Currently, this recommendation is preferable in the utilities for research (exceptionally low quality of evidence).

Comments: FeNO and blood eosinophilia plays an important role in predicting anti-inflammatory treatment outcomes of chronic cough. Currently, some non-RCT clinical trials implied that $\mathrm{FeNO}$ has a predictive value in diagnosis and treatment response of corticosteroids responsive cough, including CVA and EB. A meta-analysis of observational studies to report the diagnostic utility of FeNO for cough variant asthma and eosinophilic bronchitis shows, FeNO measurement had a moderate diagnostic accuracy in predicting CVA in patients with chronic cough, but the diagnostic accuracy to predict EB was found to be relatively lower (12). Considering a lack of high-quality RCTs, therefore, this guideline offered limited recommendations on predictive threshold of FeNO levels in patients with chronic cough. In terms of the management of chronic cough patients with high FeNO or elevated blood eosinophils, corticosteroids and antileukotriene therapy are recommended as the first option. The current investigations on anti-leukotriene treatment are non-RCT trials with insufficient clinical samples and short-term treatment duration. The FeNO values of greater than $50 \mathrm{ppb}$ were advocated by the earliest ATS guideline to indicate a possible existence of eosinophilic inflammation and a sensitive response to corticosteroid treatment. But the reference range of FeNO levels were considered too high. Multiple studies have demonstrated that the diagnosis accuracy of eosinophilic inflammation could reach $90 \%$ while the patients' FeNO values range between 30 and $40 \mathrm{ppb}$ (2). This finding could also hint that those patients might be most likely to be sensitive to corticosteroid treatment. Our research showed that with the cutoff point of 31.5 , the specificity was $91.4 \%$ to diagnose chronic cough due to eosinophilic inflammation while the sensitivity seemed much lower and presented at 54.0\% (13). What's more, a blood eosinophilic count is also regarded as a validated predictor of COPD patients' treatment response to ICS (14). To date, there is a still lack of high-quality evidence on guiding corticosteroids and anti-leukotriene treatment in patients with chronic cough.

\section{Question 3: Should anti-asthmatic drugs (anti- inflammatory or bronchodilator drugs) be used to treat patients with chronic cough?}

Recommendation 3a: We suggest a short-term inhaled corticosteroids (ICS) trial (2-4 weeks) in adult patients with chronic cough (conditional recommendation, low quality evidence).

Recommendation 3b: We suggest a short-term ICS trial (2-4 weeks) in children with chronic dry cough (conditional recommendation, low quality evidence).

Recommendation 3c: We suggest a short-term antileukotriene trial (2-4 weeks) in adults with chronic cough, particularly for those with asthmatic cough (conditional recommendation, low quality evidence).

Recommendation 3d: We suggest a short-term trial (2-4 weeks) of ICS and long-acting bronchodilator combination in adults with chronic cough and fixed airflow obstruction (conditional recommendation, moderate quality evidence).

Comments: Effectiveness of anti-asthmatic drugs (ICS and bronchodilator) was demonstrated in subjects with cough variant asthma (CVA), eosinophilic bronchitis (EB) and atopic cough (AC). However, anti-asthmatic treatment did not effectively improve cough in all patients with chronic cough. Besides ICS, some therapeutic trials proved an efficacy of leukotriene receptor antagonists in subjects with chronic cough, particularly in those with CVA and EB (15-17). Some reports showed a response to leukotriene inhibitors in patients with AC $(2,11)$. However, there are still very few RCTs with anti-leukotrienes or bronchodilators in patients with chronic cough. Combined treatment with long-acting bronchodilators is not always necessary for patients with EB and AC. We suggest targeted treatment should be prescribed after identifying the definite causes of chronic cough. If it is unavailable to perform auxiliary examinations on patients, consequently, empirical treatment regimens should be based on the recommendations mentioned above.

The ERS guideline proposed a short-term empirical therapy (2-4 weeks) of ICS. If the patients obtain satisfied effect, they can remain the original therapeutic regimens of ICS in 2-4 weeks. After 3-month empirical therapy, physicians should consider withdrawing the drug usage or reduce the drug dose. The strategy to adjust doses 
remains unclear. And it still needs further confirmation whether symptoms or inflammatory indexes are the first considerations to adjust therapies.

The application of methacholine challenge test in chronic cough remain controversial. Both CTS and ACCP suggest that it is necessary to perform methacholine challenge test to determine airway hyperresponsiveness in patients with chronic cough. The ERS guideline does not suggest the acetylcholine stimulation test as a routine test, but considers the negative acetylcholine stimulation test to be of higher guiding value. However, CTS, ACCP and ERS all recommend that, even if patients with chronic cough have airway hyperresponsiveness, the diagnosis of CVA can only be made if the asthma treatment is effective.

Even though wheezing and breathlessness don't occur in patients with chronic cough due to CVA and EB, they also present eosinophilic airway inflammation similar to those with classic asthma $(18,19)$. In clinical practice, some cases reported that symptoms easily reoccurred after withdrawing drugs, indicating 3-month etiological treatment seems not effective enough.

Furthermore, the updated ERS guideline did not highlight a combination of ICS and LABA because previous reports mainly focused on classic asthma whose end point is not the symptom of cough. In terms of the treatment for CVA, clinical trials documented evidence of significant benefits and faster onset time of combination treatment in treating CVA $(20,21)$. But the difference of ICS monotherapy and combination treatment of ICS and LABA should be deciphered in the future studies.

\section{Question 4: Should anti-acid drugs (PPIs and $\mathrm{H} 2$ antagonists) be used to treat patients with chronic cough?}

Recommendation 4: We suggest that clinicians do not routinely use anti-acid drugs in adult patients with chronic cough (conditional recommendation, low quality evidence).

Comments: Anti-acid drugs have no therapeutic effects on other common causes of chronic cough compared to gastroesophageal reflux-induced chronic cough (GERC). Thus, we don't suggest anti-acid therapy, such as proton pump inhibitor should be routinely prescribed in adult patients with chronic cough. This guideline intended for regarding all different etiologies of chronic cough as a whole, for example, proposing whether anti-asthmatic drugs (anti-inflammation drugs and bronchodilators) should be used to treat the patients with chronic cough. Based on the cough hypersensitivity underlying chronic cough, it was reasonable that different causes of chronic cough were integrated in the guideline. However, in terms of treatment regimes, a specific drug or a type of treatment option is unlikely to be useful in all the patients with chronic cough since cough resolutions depend upon the effective etiology-targeted therapy. As for whether PPIs and $\mathrm{H} 2$ antagonists should be recommended as the standard treatment for patients with GERC, ERS guideline and ACCP guideline gave out a similar recommendation that PPIs and $\mathrm{H} 2$ antagonists can be used for the patients with chronic cough co-existing with peptic symptoms, like heartburn and regurgitation (4). But, generally speaking, the expert consensus of this recommendation is relatively negative because the recommended treatments are merely targeted at a small subset of patients with GERC caused by acid reflux and, limited efficacy of anti-acid treatment is achieved. The therapeutic effect of anti-acids on GERC has been controversial, and several meta-analysis of randomized clinical trials gave out inconsistent and contradictory conclusions $(22,23)$. The main reason is that unlike the direct association of GERC and peptic syndromes, cough, as an extraesophageal symptom of gastroesophageal reflux disease, is hard to be established a clear cause-effect relationship with GERC. Our and other clinical studies have shown that classic reflux-associated symptoms, such as regurgitation and heartburn, are not always presented in the patients with GERC. And a considerable number of the patients (40-68\%) with GERC do not have classic reflux-associated symptoms but may respond favorably to medical anti-reflux therapy instead of anti-acid drugs, such as proton pump inhibitors (PPIs) alone or in combination with prokinetic agents (24-26). Therefore, cough can be effectively resolved if the decision-making of using anti-acid drugs completely depends upon the presence of concomitant peptic symptoms. Except for the attempt of Chinese cough guidelines, any other international cough guidelines haven't set up the diagnostic criteria of GERC yet $(4,10,11)$. So far, the inclusion criteria have differed in different clinical trials regarding the efficacy of anti-acid drugs on chronic cough. The sample sizes of these trials were relatively small and, most of participants did not receive the esophageal impedance-pH monitoring in diagnostic procedure. Therefore, these trials might not reflect the real enrollment situation of identified patients with GERC. There were some biases between the clinical practice and the conclusion generating from these defectively-designed trials $(27,28)$. Moreover, the therapeutic effects of anti-acid drugs are to reduce the acidity and volume of refluxates, 
ameliorate the stimulation of gastric acid or pepsin to esophageal or laryngopharyngeal mucosa, and relieve cough by the blockade of micro-aspiration and esophagealbronchial reflex (5). However, the anti-acid drugs have no effects on reducing the frequency and time of reflux episodes as well as non-acid reflux. Sometimes, they fail to achieve an ideal therapeutic outcome. At present, antiacid drugs are the second-best choice in the absence of effective options to eliminate abnormal gastroesophageal reflux, and thus, this medication should not be abolished. In the several international guidelines for the diagnosis and treatment of gastroesophageal reflux disease, antiacid drugs are still recommended as the first-line choice of treatment (29). Guided by the schematic flowchart of ACCP guideline, PPIs are also recommended as the main therapy for suspected GERC patients. As a special type of gastroesophageal reflux disease, PPIs are theoretically expected to be effective for GERC when considering their benefits in the treatment of gastroesophageal reflux disease. Several clinical studies have demonstrated that anti-acid drugs such as PPIs can alleviate the cough symptom in most of patients with GERC, supporting the application of antiacid drugs in clinical practice $(30,31)$. In the future, the benefits of anti-acid drugs on GERC need to be further validated by well-designed randomized clinical trials.

\section{Question 5: Should drugs with pro-motility activity (reflux inbibitors, prokinetics and macrolides with pro-motility activity) be used to treat patients with chronic cough?}

Recommendation 5: There is currently insufficient evidence to recommend the routine use of macrolide therapy in chronic cough. A one-month trial of macrolides can be considered in the cough of chronic bronchitis refractory instead of other therapies, taking into account local guidelines on antimicrobial stewardship (conditional recommendation, low quality evidence).

Comments: In recent years, the role of esophageal dysmotility in the pathogenesis of GERC has been gradually recognized (32). However, there is a lack of evidence-based research to demonstrate that metoclopramide, domperidone and mosapride are able to effectively alleviate the symptoms of GERC (33). Although macrolides are pro-motility drugs, they are seldom used as a prokinetic agent to treat GERC. Macrolides have once been used to treat cough related to chronic airway diseases (asthma or COPD) as the nonspecific airway anti-inflammatory drugs. However, it's questioned that macrolides are recommended to treat chronic cough, especially to manage cough symptom in the patients with chronic bronchitis as the pro-motility drugs.

\section{Question 6: Which cough neuromodulatory agents (pregabalin, gabapentin, tricyclics, and opiates) should be used to treat patients with chronic cough?}

Recommendation 6a: We recommend a trial of low dose morphine $(5-10 \mathrm{mg}$ bd) in adult patients with chronic refractory cough (strong recommendation, moderate quality evidence).

Comments: Morphine has a centrally acting antitussive effect. It is only recommended at a low dose $(5-10 \mathrm{mg}$, bd) with limited side effects. And this therapy should be stopped while there is no rapid response to morphine. In a single randomized clinical trial with a small sample size, low-dose sustained-release morphine $(5-10 \mathrm{mg}$, bd) was confirmed to significantly relieve the symptoms in adults with chronic refractory cough and improve the patients' quality of life (34). However, considering of the drug addiction as well as the worldwide strict control and management by Food and Drug Administration, morphine is unfeasibly implemented in China and many other countries even though it is strongly recommended. Furthermore, morphine is not recommended as a usual remedy for chronic refractory cough in the ACCP and Chinese guidelines $(3,11)$. Since morphine therapy targets at the symptoms rather than the etiologies of chronic cough, the patients will inevitably experience the cough recurrence after the treatment withdrawal. However, it is useful for morphine to relieve severe cough as a temporary regimen.

Codeine has an obvious antitussive effect, but the use of codeine still exists in controversy. In ERS guideline, codeine is generally not recommended due to interindividual genetic variability in drug metabolism (CYP2D6) and consequent less predictable treatment response and side-effect profile, particularly in children. CTS guideline suggest that the temporary use of codeine might be considered in severe coughs that have failed other treatments. Codeine is also contraindicated in children under 18 in China.

The value of neuromodulators has been gradually recognized in the treatment of chronic refractory cough. As a new choice for the treatment of chronic refractory cough supported by several high-quality clinical evidences $(35,36)$, neuromodulators are also recommended in ACCP and Chinese guidelines $(3,11)$. Neuromodulators (such as gabapentin or pregabalin) have antitussive effects in some patients with chronic refractory cough. The limitation of 
neuromodulators is the high frequency of adverse drug reactions. So, the patients need a close follow-up when treated with neuromodulators because the utilization of neuromodulators as an off-label drug does not benefit all the patients and will induce some central side effects, such as dizziness and drowsiness. Incremental increases in neuromodulators dose will avoid the severe adverse effects.

\section{Question 7: Should non-pharmacological therapy (cough control therapy) be used to treat patients with chronic cough?}

Recommendation 7: We suggest a trial of cough control therapy in adult patients with chronic cough (conditional recommendation, moderate quality evidence).

Comment: Non-pharmacological therapy is a novel therapy for chronic cough in recent years. There are two randomized clinical controlled trials documented evidence for its benefits $(37,38)$. When combined with drug therapy, its therapeutic efficacy can be evidently amplified (39). At present, the application of cough control treatment faces the problem of insufficient individuals qualified to perform cough control treatment, so these physical therapies have not been routinely performed. In the future, it is necessary to evaluate their merits of application in a larger group of patients from the different countries and regions and promote it.

Question 8: Should a trial of antibiotics be used in children with chronic wet cough with normal chest $X$-ray, normal spirometry, and no warning signs?

Recommendation 8: We suggest a trial of antibiotics in children with chronic wet cough with normal chest X-rays, normal spirometry, and no warning signs (conditional recommendation, low quality evidence).

Remarks: The ACCP guideline has also maintained the same recommendations (40). Since the empirical use of antibiotics is relatively common in China, the proportion of protracted bacterial bronchitis (PBB) seems not high in children with chronic cough (40). However, this recommendation is helpful to minimize the unreasonable applications of antibiotics in children.

\section{Assessment flow chart of chronic cough in children and adults recommended in ERS guideline}

Comments: The current major guidelines of chronic cough do differ in the concept and scope. ERS is characterized by (I) it views chronic cough as a clinical syndrome but not as a symptom of other diseases and that (II) it suggests identifying cough phenotypes or treatable traits rather than causes of cough. While ACCP and CTS interpret the management of chronic cough according to different causes. Due to the lack of diagnostic criteria of the common causes of chronic cough in ERS guideline, the flow charts are different from those in ACCP and Chinese guidelines. In particular, they do not require to give out the assessment of chronic cough in adults and children and specifically targeting therapy based on the definitive diagnosis of the etiologies of chronic cough, but only consider additional methods of laboratory examinations to determine the causes after the initial empirical treatment fails to resolve cough $(1,11)$.

The diagnostic protocols are feasible for the physicians in the primary or secondary care clinics, but can only be partially accepted and still need to be adjusted in the specialized cough centers according to the real conditions of available equipment and technique. In the flow chart of ERS guideline, PPIs should be only initiated when peptic symptoms or evidence of acid reflux are present. But the recommendation indicated that the therapeutic effects of anti-acid drugs for GERC have been partially agreed. The esophageal high-resolution manometry (HRM) was firstly included in the diagnostic protocol of chronic cough, which reflects a new progress and understanding of esophageal dysmotility underlying the development of GERC (29). However, it remains a doubt that esophageal impedance-pH monitoring is not recommended to apply, which is inconsistent with the normal diagnostic modality of gastroesophageal reflux disease adopted by the gastroenterologists. The efficiency of the simple $24 \mathrm{~h}$ esophageal $\mathrm{pH}$ monitoring on diagnosing GERC is limited. But combined with esophageal impedance [multichannel intraluminal impedance-pH monitoring (MII$\mathrm{pH})$ ] or even HRM probably improve the accuracy of diagnosis. MII-pH can detect abnormal acid and non-acid reflux in the esophagus and analyze the association between reflux and cough (41). At present, MII-pH is regarded as a sensitive and specific test for diagnosing GERC and recommended by ACCP and CTS guideline $(1,11)$. HRM can identify oesophageal dysmotility and potentially provide a diagnosis in patients with unexplained cough $(41,42)$. Additionally, HRM is helpful to determine the position of lower esophageal sphincter for accurately placing $\mathrm{pH}$ impedance electrode catheters (41). Therefore, esophageal 
$\mathrm{pH}$ monitoring combines with esophageal impedance and even HRM may be helpful for the diagnosis of GERC. In addition, the etiologies of chronic cough are complicated. In clinical practice, uncommon or rare causes also need attention after consideration of the common etiologies. As CTS guideline summarized, there are varieties of uncommon causes of chronic cough such as bronchial foreign body, tracheobronchial amyloidosis, pulmonary alveolar proteinosis, etc. Sometimes, the management of uncommon or rare chronic cough etiologies may need attention in clinical practice.

In general, the updated ERS guideline for the management of chronic cough has a concise structure and comprehensive content with several concepts updated. In particular, according to PICO framework and the GRADE approach, the direction-oriented and practical corresponding recommendations have been formulated and graded to address some key issues of clinical concern for cough management raised in recent years. However, we also noticed that some literatures cited in the recommendations are not of high quality and, the solution devised for some questions are still controversial or inconclusive. Since there is a lack of diagnostic criteria for the causes of chronic cough, some recommendations for the treatment specific to the etiologies still remain ambiguous. Under the condition of empirical treatment, the applicability of the diagnosis and treatment pathways related to clinical management needs to be further evaluated. A simple pragmatic pathway probably improved by further discovery and validation of practical biomarkers should be introduced for managing chronic cough. Therefore, the clinical application of the new ERS guideline is necessary to be further evaluated with the combination of clinical practice in China. Additionally, more collaborations with worldwide cough specialists are needed to accelerate the progress of clinical research and to provide more solid evidence for updating cough guidelines. As a common major health issue, whether chronic cough should be regarded as a disease entity in the international disease classification system deserves further discussion.

\section{Acknowledgments}

Funding: None.

\section{Footnote}

Provenance and Peer Review: This article was commissioned by Dr. Alyn H. Morice for the series "Cough Section" published in Fournal of Thoracic Disease. The article was sent for external peer review organized by the Guest Editor Dr. Woo-Jung Song, Dr. Alyn H. Morice and the editorial office.

Conflicts of Interest: All authors have completed the ICMJE uniform disclosure form (available at http://dx.doi. org/10.21037/jtd-2020-065). Dr. KL serves as an unpaid editorial board member of Fournal of Thoracic Disease. The authors have no other conflicts of interest to declare.

Ethical Statement: The authors are accountable for all aspects of the work in ensuring that questions related to the accuracy or integrity of any part of the work are appropriately investigated and resolved.

Open Access Statement: This is an Open Access article distributed in accordance with the Creative Commons Attribution-NonCommercial-NoDerivs 4.0 International License (CC BY-NC-ND 4.0), which permits the noncommercial replication and distribution of the article with the strict proviso that no changes or edits are made and the original work is properly cited (including links to both the formal publication through the relevant DOI and the license). See: https://creativecommons.org/licenses/by-nc-nd/4.0/.

\section{References}

1. Irwin RS, French CL, Chang AB, et al. Classification of Cough as a Symptom in Adults and Management Algorithms: CHEST Guideline and Expert Panel Report. Chest 2018;153:196-209.

2. Côté A, Russell RJ, Boulet LP, et al. Managing Chronic Cough Due to Asthma and NAEB in Adults and Adolescents: CHEST Guideline and Expert Panel Report. Chest 2020;158:68-96.

3. Gibson P, Wang G, McGarvey L, et al. Treatment of Unexplained Chronic Cough: CHEST Guideline and Expert Panel Report. Chest 2016;149:27-44.

4. Kahrilas PJ, Altman KW, Chang AB, et al. Chronic Cough Due to Gastroesophageal Reflux in Adults: CHEST Guideline and Expert Panel Report. Chest 2016;150:1341-60.

5. Irwin RS. Chronic cough due to gastroesophageal reflux disease: ACCP evidence-based clinical practice guidelines. Chest 2006;129:80S-94S.

6. Chang AB, Oppenheimer JJ, Irwin RS, et al. Managing Chronic Cough as a Symptom in Children and Management Algorithms: CHEST Guideline and Expert 
Panel Report. Chest 2020;158:303-29.

7. McGarvey LP, Heaney LG, Lawson JT, et al. Evaluation and outcome of patients with chronic non-productive cough using a comprehensive diagnostic protocol. Thorax 1998;53:738-43.

8. Berrington de González A, Mahesh M, Kim KP, et al. Projected cancer risks from computed tomographic scans performed in the United States in 2007. Arch Intern Med 2009;169:2071-7.

9. Brenner DJ, Hall EJ. Computed tomography--an increasing source of radiation exposure. $\mathrm{N}$ Engl J Med 2007;357:2277-84.

10. Morice AH, Millqvist E, Bieksiene K, et al. ERS guidelines on the diagnosis and treatment of chronic cough in adults and children. Eur Respir J 2020;5 5:1901136.

11. Lai K, Shen H, Zhou X, et al. Clinical Practice Guidelines for Diagnosis and Management of Cough-Chinese Thoracic Society (CTS) Asthma Consortium. J Thorac Dis 2018;10:6314-51.

12. Song WJ, Kim HJ, Shim JS, et al. Diagnostic accuracy of fractional exhaled nitric oxide measurement in predicting cough-variant asthma and eosinophilic bronchitis in adults with chronic cough: A systematic review and meta-analysis. J Allergy Clin Immunol 2017;140:701-9.

13. Yi F, Chen R, Luo W, et al. Validity of Fractional Exhaled Nitric Oxide in Diagnosis of Corticosteroid-Responsive Cough. Chest 2016;149:1042-51.

14. Calverley P, Pauwels R, Vestbo J, et al. Combined salmeterol and fluticasone in the treatment of chronic obstructive pulmonary disease: a randomised controlled trial. Lancet 2003;361:449-56.

15. Bao W, Liu P, Qiu Z, et al. Efficacy of add-on montelukast in nonasthmatic eosinophilic bronchitis: the additive effect on airway inflammation, cough and life quality. Chin Med J (Engl) 2015;128:39-45.

16. Dicpinigaitis PV, Dobkin JB, Reichel J. Antitussive effect of the leukotriene receptor antagonist zafirlukast in subjects with cough-variant asthma. J Asthma 2002;39:291-7.

17. Spector SL, Tan RA. Effectiveness of montelukast in the treatment of cough variant asthma. Ann Allergy Asthma Immunol 2004;93:232-6.

18. Brightling CE, Bradding P, Symon FA, et al. Mast-cell infiltration of airway smooth muscle in asthma. N Engl J Med 2002;346:1699-705.

19. Yoo Y, Koh YY, Kang H, et al. Sputum eosinophil counts and eosinophil cationic protein levels in cough-variant asthma and in classic asthma, and their relationships to airway hypersensitivity or maximal airway response to methacholine. Allergy 2004;59:1055-62.

20. Bao W, Chen Q, Lin Y, et al. Efficacy of procaterol combined with inhaled budesonide for treatment of coughvariant asthma. Respirology 2013;18 Suppl 3:53-61.

21. Tagaya E, Kondo M, Kirishi S, et al. Effects of regular treatment with combination of salmeterol/fluticasone propionate and salmeterol alone in cough variant asthma. J Asthma 2015;52:512-8.

22. Chang AB, Lasserson TJ, Gaffney J, et al. Gastrooesophageal reflux treatment for prolonged non-specific cough in children and adults. Cochrane Database Syst Rev 2011;2011:CD004823.

23. Kahrilas PJ, Howden CW, Hughes N, et al. Response of chronic cough to acid-suppressive therapy in patients with gastroesophageal reflux disease. Chest 2013;143:605-12.

24. Irwin RS, Madison JM. Anatomical diagnostic protocol in evaluating chronic cough with specific reference to gastroesophageal reflux disease. Am J Med 2000;108 Suppl 4a:126S-30S.

25. Lai KF, Chen RC, Lin L, et al. Diagnostic values of the clinical characteristics of chronic cough. Zhonghua Jie He He Hu Xi Za Zhi 2009;32:418-21.

26. Xu X, Yang Z, Chen Q, et al. Comparison of clinical characteristics of chronic cough due to non-acid and acid gastroesophageal reflux. Clin Respir J 2015;9:196-202.

27. Faruqi S, Molyneux ID, Fathi H, et al. Chronic cough and esomeprazole: a double-blind placebo-controlled parallel study. Respirology 2011;16:1150-6.

28. Shaheen NJ, Crockett SD, Bright SD, et al. Randomised clinical trial: high-dose acid suppression for chronic cough - a double-blind, placebo-controlled study. Aliment Pharmacol Ther 2011;33:225-34.

29. Gyawali CP, Kahrilas PJ, Savarino E, et al. Modern diagnosis of GERD: the Lyon Consensus. Gut 2018;67:1351-62.

30. Qiu Z, Yu L, Xu S, et al. Cough reflex sensitivity and airway inflammation in patients with chronic cough due to non-acid gastro-oesophageal reflux. Respirology 2011;16:645-52.

31. Xu X, Chen Q, Liang S, et al. Comparison of gastroesophageal reflux disease questionnaire and multichannel intraluminal impedance $\mathrm{pH}$ monitoring in identifying patients with chronic cough responsive to antireflux therapy. Chest 2014;145:1264-70.

32. Bennett MC, Patel A, Sainani N, et al. Chronic Cough Is Associated With Long Breaks in Esophageal Peristaltic Integrity on High-resolution Manometry. J Neurogastroenterol Motil 2018;24:387-94. 
33. Hillman L, Yadlapati R, Thuluvath AJ, Berendsen MA, Pandolfino JE. A review of medical therapy for proton pump inhibitor nonresponsive gastroesophageal reflux disease. Dis Esophagus 2017;30:1-15.

34. Morice AH, Menon MS, Mulrennan SA, et al. Opiate therapy in chronic cough. Am J Respir Crit Care Med 2007;175:312-5.

35. Bowen AJ, Nowacki AS, Contrera K, et al. Short- and Long-term Effects of Neuromodulators for Unexplained Chronic Cough. Otolaryngol Head Neck Surg 2018;159:508-15.

36. Dong $\mathrm{R}, \mathrm{Xu} \mathrm{X}, \mathrm{Yu} \mathrm{L}$, et al. Randomised clinical trial: gabapentin vs baclofen in the treatment of suspected refractory gastro-oesophageal reflux-induced chronic cough. Aliment Pharmacol Ther 2019;49:714-22.

37. Chamberlain Mitchell SAF, Garrod R, Clark L, et al. Physiotherapy, and speech and language therapy intervention for patients with refractory chronic cough: a multicentre randomised control trial. Thorax 2017;72:129-36.

Cite this article as: Chen R, Qiu Z, Lai K. 2019 ERS cough guideline: consensus and controversy. J Thorac Dis 2020;12(12):7504-7514. doi: 10.21037/jtd-2020-065
38. Vertigan AE, Theodoros DG, Gibson PG, et al. Efficacy of speech pathology management for chronic cough: a randomised placebo controlled trial of treatment efficacy. Thorax 2006;61:1065-9.

39. Vertigan AE, Kapela SL, Ryan NM, et al. Pregabalin and Speech Pathology Combination Therapy for Refractory Chronic Cough: A Randomized Controlled Trial. Chest 2016;149:639-48.

40. Chang AB, Oppenheimer JJ, Weinberger MM, et al. Management of Children With Chronic Wet Cough and Protracted Bacterial Bronchitis: CHEST Guideline and Expert Panel Report. Chest 2017;151:884-90.

41. Kahrilas PJ, Sifrim D. High-resolution manometry and impedance-pH/manometry: valuable tools in clinical and investigational esophagology. Gastroenterology 2008;135:756-69.

42. Burke JM, Jackson W, Morice AH. The role of high resolution oesophageal manometry in occult respiratory symptoms. Respir Med 2018;138:47-9. 